\title{
Pour un système de santé plus efficace
}

Judith Trageser, Anna Vettori, Rolf Iten, Juliane Fliedner

INFRAS

L'étude, mandatée par l'Association des entreprises pharmaceutiques en Suisse (vips), a bénéficié de l'accompagnement d'un groupe d'expert-e-s composé de Felix Gutzwiller (Conseiller aux États) Peter Indra (Département de la santé publique, Bâle-Ville), Urs Meister (Avenir Suisse), Sandra Schneider (Office fédéral de la santé publique) et de Jean-François Steiert (Conseiller national).

\begin{abstract}
Le système de santé suisse serait plus efficace si l'on s'inspirait d'une série d'approches pratiquées à l'étranger; produire une série d'incitations du côté de l'offre et fournir les preuves de leur efficacité permettrait à diffuser ces approches. Telles sont les conclusions de l'étude d'INFRAS qui a analysé des approches choisies. L'étude présente également leur potentiel d'efficacité et propose des solutions pour une mise en œuvre efficace en Suisse.
\end{abstract}

\section{De l'inefficacité du système de santé suisse}

«Le système de santé suisse? Bon, mais coûteux.» Tel est le bilan, généralement reconnu. Une série d'études montre toutefois que l'efficacité de ce système, dont la qualité élevée est indiscutable, peut encore être améliorée. Aujourd'hui, le manque d'efficacité qui se manifeste dans certains domaines précis, comme par exemple dans le manque de coordination au niveau des soins, dans la surabondance de prestations non indispensables et dans une demande excessive de la part des personnes assurées, représente pas moins de 10 pour cent de l'ensemble des dépenses de santé annuelles [1]. Le Conseil fédéral, de son côté, estime dans sa stratégie "Santé 2020" qu'en accroissant l'efficacité du système de santé, on pourra économiser 20 pour cent des coûts.

Compte tenu de ces prémisses, l'étude INFRAS* intitulée «Pour un système de santé plus efficace. Sélection d'approches» [2] analyse une série de réorientations aptes à réduire les causes des inefficacités les plus marquées. L'étude se concentre sur des solutions

Incitations au niveau de l'offre
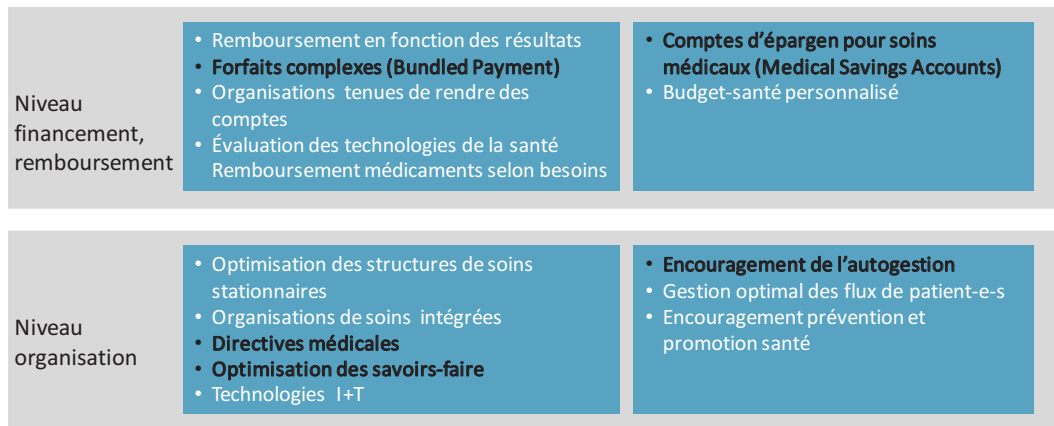

Figure 1: Typologie des solutions envisageables. qui ont fait leurs preuves à l'étranger et qui ne sont encore que peu répandues en Suisse.

\section{Les solutions envisageables: coup d'œil au-delà des frontières nationales}

A l'étranger, de nombreuses approches sont mises à l'épreuve avant d'être appliquées avec succès en vue de réduire l'inefficacité des systèmes de santé. Elles cherchent notamment à accroître la transparence en matière de qualité des prestations fournies, à centrer les soins sur les patient-e-s en mettant en place des organisations intégrées, à associer activement les patient-e-s à leurs soins et à renforcer les structures d'approvisionnement du secteur ambulatoire.

Dans le cadre de l'étude INFRAS, quinze approches qui ont pour but d'améliorer l'efficacité du système de santé ont été identifiées; elles interviennent à divers niveaux du système et proposent divers types d'incitations (fig. 1).

Dans le cadre de l'étude INFRAS, cinq des approches présentées à la figure 1 ont été sélectionnées pour être approfondies (en noir). Cet approfondissement, qui se base sur l'étude de la littérature spécialisée, a mis en lumière le potentiel d'efficacité de chacune de ces cinq approches. Par ailleurs, il s'agissait d'établir si ces approches pouvaient être introduites ou propagées en Suisse; des interviews ont été menées dans ce but avec les différentes parties prenantes.

\section{Les forfaits complexes}

Par forfaits complexes (Bundled Payment), on entend un modèle de remboursement forfaitaire pour l'ensemble des prestations fournies durant une période 
de soins par les divers prestataires (par ex. médecin du secteur ambulatoire, hôpital, réhabilitation, physiothérapie. Le remboursement forfaitaire incite les prestataires de soins à optimiser le nombre et le coût des prestations fournies durant un épisode de maladie et à coordonner de manière optimale ces différentes prestations. Sur la base des expériences faites avec ce modèle à l'étranger, on peut partir de l'idée que le modèle des forfaits complexes constitue une solution apte à améliorer la qualité, les résultats (c'est-à-dire l'état de santé) et le rapport coût-efficacité du système de santé suisse. Selon une estimation succincte, le modèle des forfaits complexes permet d'économiser 650 millions de francs par an (soit 1 pour cent des dépenses de santé) dans les seuls domaines du remplacement des articulations du genou et des hanches, de l'infarctus du myocarde et du diabète. Toutefois, ce modèle comporte quelques risques, tels que le non-recours à des prestations appropriées ou une bureaucratie importante.

Que sont les forfaits complexes?

Le remboursement forfaitaire d'un ensemble de prestations liées à un épisode de maladie.

- Objectif: améliorer la coordination entre les prestataires de soins; diminution de la demande induite par l'offre.

Où pratique-t-on ce système?

- USA, NL, SE, UK: affections chroniques, épisodes aigus.

- En Suisse: application de modèles isolés, dans le domaine des soins aigus (essentiellement en orthopédie).

Quels sont les obstacles en Suisse?

- Manque d'incitations, réserves, investissement requis de la part du corps médical

- Système de soins fragmenté

- Absence de données, infrastructure technique insuffisante

- Nombre de cas faible, et par conséquent dispersion des risques au niveau des forfaits

Quels sont les facteurs encourageants?

- Changements structurels dans les hôpitaux (collaborations plus étroites avec le secteur ambulatoire)

- Stratégie Cybersanté Suisse

- Amélioration de la compensation des risques (nombre accru d'incitations pour les assureurs)

\section{La combinaison optimale des savoir-faire}

La combinaison optimale des savoir-faire (Skill Mix) a pour but de redistribuer le travail et les compétences entre les professions de la santé, de manière à ce que les prestations puissent être fournies avec un minimum de ressources, tout en maintenant un niveau qualitatif au moins égal au niveau actuel. Selon les preuves empiriques disponibles pour le domaine du personnel soignant et des médecins, l'optimisation obtenue par la combinaison optimale des savoir-faire est essentiellement sensible au niveau de la qualité de l'approvisionnement, de la satisfaction des patient-e-s et de l'état de santé. Les preuves sont moins claires en termes de baisse des coûts, notamment parce que ce modèle comporte le risque de multiplier le nombre des prestations.

Qu'est-ce que la combinaison optimale des savoir-faire?

Redistribution du travail et des compétences entre les professionnel-le-s de la santé, en l'occurrence entre les médecins de premier recours et les soignant-e-s.

- Objectif: diminution des ressources et maintien de la qualité; endiguement de la pénurie de main d'œuvre.

Où pratique-t-on ce système?

- A l'étranger: modèle très répandu, en particulier dans les pays anglo-saxons (infirmières/infirmiers en soins avancés APN, cliniques gérées par des infirmières/infirmiers, etc.)

- En Suisse: recours aux infirmières/infirmiers en soins avancés dans les hôpitaux, exemples isolés dans

l'approvisionnement de base; quelques projets pilotes.

Quels sont les obstacles en Suisse?

- Réticences du corps médical à propos de la qualité

- Non-compensation de prestations par le système tarifaire

- Système de santé fragmenté

- Carences légales (p. ex. responsabilité civile)

- Système de formation professionnelle peu axée sur

I'interprofessionnalité

Quels sont les facteurs encourageants?

- Pénurie de main d'œuvre au niveau des soins de base et des soins en général

- Meilleure acceptation suite à des essais pilotes concluants

- Forfaits (DRG, forfaits par patient-e) et par conséquent pression pour améliorer le rapport coût-efficacité

- Stratégie Cybersanté Suisse

\section{Directives médicales}

Les directives médicales (Medical Guidelines) sont des recommandations basées sur des données scientifiques, destinées à optimiser et à standardiser le traitement des patient-e-s. L'objectif de telles directives est d'accroître l'efficacité des processus et d'éviter les prestations superflues ainsi que les traitements désuets. Elles contribuent donc à lutter contre le sur-approvisionnement en prestations, l'une des principales sources d'inefficacité du système de santé suisse. Dans l'ensemble, ce modèle fait ses preuves. On a ainsi démontré qu'il produit des diagnostics plus précis, que les modifications de la médication sont moins fréquentes et que les médecins réexaminent plus facilement l'opportunité des prestations. Quant à l'effet de ce modèle sur le rapport coût-utilité, il n'a été que peu étudié, car difficile à prouver. 
Que sont les directives médicales?

Recommandations pour les médecins, basées sur des données scientifiques.

- Objectif: optimisation et standardisation du traitement des patient-e-s

Où pratique-t-on ce système?

- A l'étranger: documents très répandus dans de nombreux pays et domaines médicaux; méthodologie en partie institutionnalisée, p. ex. en Allemagne

- En Suisse: présent à un stade rudimentaire dans certains domaines spécialisés (cardiologie, oncologie, diabète, etc.); élaboration par les corporations spécialisées; dans certains hôpitaux: directives internes.

Quels sont les obstacles en Suisse?

- Méconnaissance, craintes des médecins de voir restreindre leur liberté thérapeutique, craintes de sous-approvisionnement (médecins et patient-e-s)

- Efficacité insuffisamment documentée scientifiquement

- Moyens financiers insuffisants pour l'élaboration de telles directives

- Manque d'incitations dans l'approvisionnement de base

Quels sont les facteurs encourageants?

- Forfaits (DRG, forfaits par patient-e) et par conséquent pression pour améliorer le rapport coût-efficacité

- Stratégie Cybersanté Suisse

\section{Compte d'épargne pour soins médicaux}

Le compte d'épargne pour soins médicaux (Medical Savings Accounts, MSA) est un compte d'épargne individuel réservé aux soins médicaux. La personne qui détient un compte l'alimente elle-même en cotisant régulièrement. En cas de maladie, elle paie les frais de traitement en puisant dans les économies qu'elle a ainsi constituées. L'objectif du compte d'épargne pour soins médicaux est de renforcer la prise de conscience des coûts médicaux par les patient-e-s. Fréquemment, une assurance pour hauts risques complète ce compte. Les études consacrées aux effets de ce modèle ne fournissent pas de preuves irréfutables quant à son efficacité économique; les effets seraient même négatifs au niveau des coûts, notamment parce que les patient-e-s tardent à recourir aux prestations. Néanmoins, le modèle a l'avantage de décharger financièrement la jeune génération à une époque où l'évolution démographique fait grimper les coûts de santé des aînés. Les expert-e-s craignent toutefois la mise en péril du principe de solidarité.

Qu'est-ce que le compte d'épargne pour soins médicaux?

Un compte d'épargne destiné à payer les frais de maladie (exception: cas à haut risques)

- Objectif: aiguiser la prise de conscience pour le coût des

patient-e-s, renoncer aux prestations inutiles, améliorer la justice intergénérationnelle.
Où pratique-t-on ce système?

- Singapour, Chine, Afrique du Sud, USA

Quels sont les obstacles en Suisse?

- Crainte d'une désolidarisation

- Aucune preuve d'une amélioration de la qualité ou d'un

meilleur rapport coût-efficacité

- Absence de bases légales

- Message politique difficile à communiquer

Quels sont les facteurs encourageants?

- Evolution démographique; allègement du fardeau de la jeune génération

\section{Encourager l'autogestion des patient-e-s}

Par autogestion des patient-e-s, on entend l'habilitation des patient-e-s à vivre de manière autonome et indépendante. Ainsi, les programmes spécialisés proposent par exemple des informations pour malades chroniques et des entraînements personnels pour les patient-e-s. Ce modèle veut d'une part mieux adapter l'approvisionnement de soins aux besoins des patient-e-s et d'autre part juguler le recours à des prestations exigées inutilement, en raison d'un manque d'information. Divers exemples font état d'effets positifs en termes de satisfaction des patient-e-s, zd'effets sur la santé (par ex. taux de glycémie) et de taux d'hospitalisation. En même temps, la littérature spécialisée montre qu'un modèle basé sur l'observance des patient-e-s comporte un risque d'incertitude quant aux effets sur la santé.

Qu'est-ce que l'autogestion des patient-e-s?

L'aptitude des patient-e-s à gérer leur maladie.

- Objectif: éviter les complications

Où pratique-t-on ce système?

- Etranger: divers programmes très répandus p. ex. dans les pays suivants: Australie, USA, GB

- Suisse: divers programmes/projets pilotes, p. ex. cours EVIVO pour les patient-e-s, projets LEILA - Vivre avec une maladie chronique

Quels sont les obstacles en Suisse?

- Craintes d'une observance insuffisante de la part des patient-e-s

- Non-remboursement des prestations, systèmes tarifaires

- Manque d'incitations pour les assurances

Quels sont les facteurs encourageants?

- Bonne acceptance de principe

- Hausse des besoins de soins des malades chroniques

- Meilleure compensation des risques

Stratégie Cybersanté Suisse

\section{Conclusions}

- Elargissement du potentiel d'efficacité au moyen de démarches modulaires. L'analyse de cinq types de 
solutions montre qu'il n'existe pas de «géant endormi» dont le réveil permettrait d'activer d'un seul coup de grands potentiels d'efficacité. En approfondissant, on constate néanmoins que l'accroissement de l'efficacité sera obtenu à l'aide d'un ensemble de mesures qui se complètent et que le montant escompté d'économies des coûts de santé devrait se situer entre 1 et 10 pour cent.

- Il n'est pas nécessaire de bouleverser le système de santé pour faire mieux. Parmi les solutions présentées, nombreuses sont celles qui peuvent être introduites et mises en œuvre dans le système existant. C'est en particulier le cas des approches qui demandent des initiatives et des changements d'ordre organisationnel de la part des prestataires de services (par ex. combinaison optimale des savoir-faire, directives médicales). D’ailleurs, des ébauches de telles approches existent déjà en Suisse. Par conséquent, et à l'exception du modèle du compte d'épargne pour soins médicaux, aucun bouleversement fondamental du système de santé suisse n'est nécessaire pour accroître son efficacité.

- Les besoins des patient-e-s au centre de l'attention: Parmi les approches envisageables pour accroître l'efficacité, beaucoup découlent de l'idée des «soins orientés vers le résultat» (value-based care), où les besoins des patient-e-s sont au centre de l'attention. L'utilité de la prestation pour les patient-e-s constitue un moteur déterminant non seulement pour accroître la qualité du système de santé, mais aussi pour améliorer le rapport coûtefficacité.

- La mise en œuvre des potentiels d'efficacité dépend de leur formulation concrète. L'approfondissement des modèles montre que les esquisses de solutions proposées comportent certains risques et que les détails dont il faut tenir compte ne sont pas négligeables. Ce sont les dispositions concrètes qui permettent de réaliser le potentiel d'efficacité.

Judith Trageser

Infras

Forschung und Beratung

Binzstrasse 23

CH-8045 Zurich

Tél. 0442059526

judith.trageser[at]infras.ch

loir imposer des solutions "d'en haut» n'est pas une démarche convaincante, preuve en sont les exemples documentés à l'étranger. Il s'agit de mo- tiver les prestataires de services à élaborer puis à appliquer eux-mêmes des solutions prometteuses.

- Inciter à l'aide de réglementations: pour que les prestataires de services mettent en pratique de nouvelles solutions, ces dernières doivent comporter des incitations. Aujourd'hui encore, le système de santé suisse comporte des facteurs incitatifs qui vont à contre-sens, telle la fragmentation extrême de l'approvisionnement dans le pays, l'indemnisation à la prestation dans le secteur ambulatoire ou les tarifs en vigueur, qui n'affichent pas la totalité des prestations.

- Les preuves scientifiques et les exemples de bonnes pratiques, conditions de succès: malgré les succès enregistrés à l'étranger, les pistes proposées se heurtent souvent à des résistances; elles sont en partie dues au manque d'information des parties prenantes. Pour être garantes de succès, les nouvelles approches dépendent donc des preuves scientifiques dont elles sont dotées et de la promotion des bonnes pratiques.

- Le Conseil fédéral prend les devants: le Conseil fédéral a pris des mesures déterminantes pour favoriser l'implantation de nouvelles solutions. Ce sont par exemple la stratégie de la qualité, la loi sur les professions de la santé, la Stratégie Cybersanté Suisse, le perfectionnement de la compensation des risques et la multiplication des données sur les soins. Par ailleurs, la stratégie "Santé 2020" confirme que le Conseil fédéral a su reconnaître à la fois les défis principaux à relever dans le domaine du système de santé et les pistes les plus prometteuses qui permettent de les relever. Désormais, il s'agira de mettre en œuvre de manière systématique les démarches qui s'y rapportent, en impliquant toutes les milieux concernés.

Références

1 INFRAS, Crivelli. Effizienz, Nutzung und Finanzierung des Gesundheitswesens. Bern: Akademien der Wissenschaften Schweiz; 2012.
2 INFRAS 2014. Pour un système de santé plus efficace. Sélection d'approches. À télécharger sur www.infras.ch/f/projekte/ displayprojectitem.php?id=4929. 